\title{
Blockchain-based Big Data Analytics Approach for Smart Cities
}

\author{
Tanweer Alam \\ Faculty of Computer and Information Systems, Islamic University of Madinah, Saudi Arabia. \\ Email: tanweer03@iu.edu.sa
}

\begin{abstract}
The wireless communication is the fastest-growing field of studies to enable humans to communicate with each other. Introducing a new blockchain architecture with big data analytics can be an enticing platform towards increasing connectivity performance throughout the smart cities. Blockchains can build a convenient mechanism allowing two devices to effectively transmit currency as well as data, to each other with a secure and reliable agreement. Smart cities provide an opportunity to connect people and places that use emerging technologies that help to improve urban planning and development. Smart cities can enhance public infrastructure and people's living experience. Presently, high-speed, intelligent, effective with several innovations, like low energy consumption, etc., seem to be accessible to interact together in the modern environment. By using the blockchain-based big data approach the physical devices are allowed to communicate securely with other physical devices in heterogeneous environments. This approach builds a new blockchain-based computing structure throughout the IoT technology configuration. This approach can examine blockchain technology to the underlying technology or maintains the IoT authentication reliable. This strengthens blockchain and cloud to develop an enabling IoT pervasive environment for secure communication among the physical objects.
\end{abstract}

Keywords-Blockchain, Big Data Analytics, Wireless Communication, Information Security, Data transmission, Smart Cities, Internet of Things.

\section{Introduction}

Wireless communication is the key of Smart Cities. It is expected to exceed 75 billion connected devices by 2025 and most of these nodes cannot be connected by the wired network [1]. Encourages industrial applications like smart manufacturing plants or smart houses, the networking processes must address the non-deterministic essence of wireless connections [2]. This proposed research work in this project is an enhancement and implementation of the Big Data analytics framework in smart cities architecture based on blockchain technology. The results of its research develop a new mechanism. Such a study utilizes a required present study precise and effective computation and could be introduced in a smart city scope. The researchers can improve this study and implement it on the internet of everything framework in the future. Emerging Communication technologies play a key role in smart cities by providing information gathered across internet technologies. Smart Cities have the essential infrastructures to facilitate the residents including the quality way of living. Big data would have an enormous ability to expand and utilize smart city facilities [3]. The Big data can play a vital role in analyzing information obtained from the Internet of things gadgets in the smart cities, therefore, further evaluation could be done to acknowledge the techniques and demands in the smart cities [4]. The sensors deployed throughout the region produce massive amounts of data, but many changes could be made when used efficiently. It is essentially massive volumes of data that industries could evaluate to consider making suitable strategic decisions as well as business management [5]. From this huge amount of data to reveal trends and give the insight to obtain useful data. 


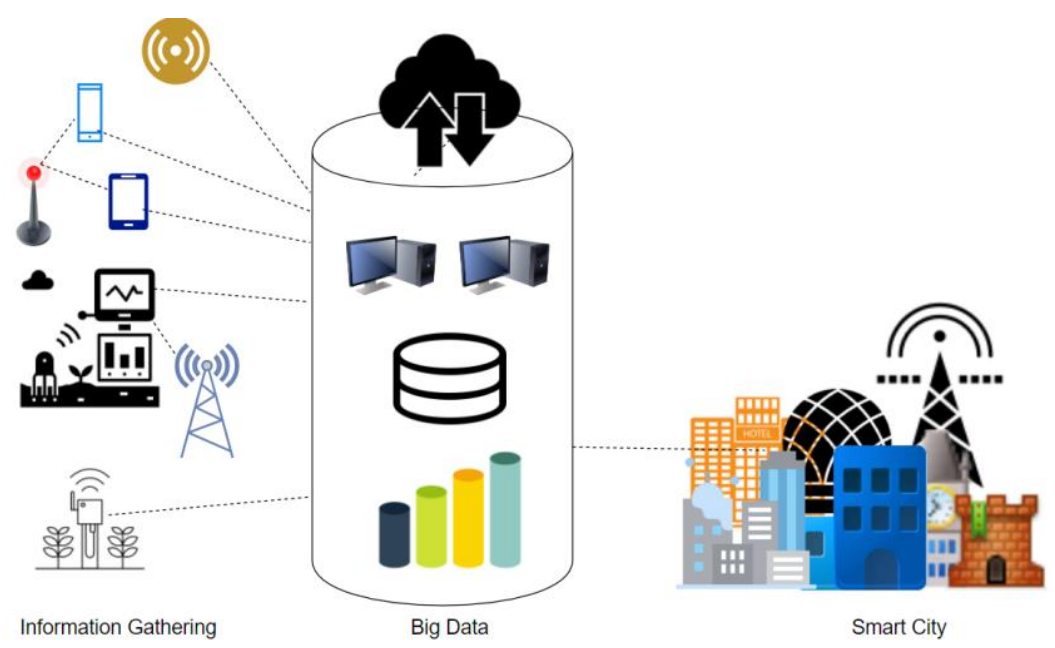

Figure 1. Information gathering and storing for smart cities

Developing a new blockchain-based Big data analytics framework in the architecture of smart cities can be a valuable framework to improve the performance of communication security in a heterogeneous environment. Blockchain is an innovation working on distributed ledger technology. The blockchain follows a decentralized manner with more securities [6]. The transactions have been stored in the blocks. Every new transaction has been stored in a new verified block. Such blocks are all interconnected with blockchain. These blocks contain huge data received from transactions. Massive blocks data containing a complete record of each transaction, that are required for evaluation purpose. The Blockchain offers ledger credibility without the evaluation. Therefore, the Big Data takes place as well as corresponding analytics techniques. The Big data is the collection of huge measurements [7]. As the data is incredibly big and complicated, the conventional data analysis technologies could not process this. Today, the demand for predictive big data analytics is high. The main reason for driving such a large market is the rather-increasing real-time Big Data programs. Municipal governments utilize cameras, infrastructure, and mobile applications to collect relevant information, like congestion, energy consumption, and air pollution. This information could then be used through technical solutions to enhance community infrastructure, covering services, transportation, and public security [8]. Figure 1 shows the data gathering and processing using Big data for smart cities.

Large transaction information is processed in various ways of ledgers online nowadays. The Blockchain could make online data processing effectively. At the highest level of that, the distributed infrastructure could give several remote accesses. The transaction data are stored in distinct databases by multiple groups in the occurrence of the transaction [9]. With the assistance of Blockchain, those groups could be provided access to a large system. The main contribution is to tie new research that embeds blockchains with big data and offers connectivity security in the smart cities. Such a study provides improved blockchain and big data to create an effective communication structure between many connected devices in smart cities. The distinction of such a study with previous examinations is depicted by data transmission limitations, validation, package width range, and declining node generation information sharing. That methodology has been implemented scientifically. A revised structure makes our extensive studies predictable excellently. Also, computational findings are validated. This approach could be helpful for upscale the quality of life of residents and visitors of Al-Madinah Al-Munawwarah.

This proposed research plan builds research on extending the communication in the internet of things using big data and blockchain technologies. Transferring data from one configuration to another using wireless network starts from 1973 in the form of packets radio network techniques [10]. These configurations were able to communicate with another same configuration system. The latest work is continuing a project called the Serval Project study. This offers facilities for smart devices to communicate in infrastructure-less 
platforms. However, our research is concerned about communication security and performance among the smart devices in the smart city.

The key contribution of this research is the creation of the communication framework and provide reliable and fast communication using big data and blockchain among the internet of smart devices for smart cities. Previous studies have been focused on the creation and optimization of the framework for communication, but such research does not perform the full framework for secure communication among the internet of smart devices using blockchain and big data. The research questions are 1) How will the transaction data secure in smart cities? 2) How will the data access in real-time? The following are the key points in this research-

1 This research is primarily focused on smart cities. This allows connected devices to interact between smart gadgets utilizing blockchain technology and big data in the smart cities.

2 This framework for communication will access across the internet of smart devices in the smart cities.

The rest of the paper is divided into the following sections. Sect. 2 shows the related works, Sect. 3 represents the possibilities for the proposed integration approach, Sect. 4 shows the methodology and results, Sect. 5 shows the discussion, Sect. 6 represents the applications, and sect. 7 represents the conclusion and future scopes of the proposed approach.

\section{Related works}

In 2016, Mayra Samaniego et.al. were published an article on "Blockchain as a service for IoT". In this article, they have presented the blockchains as a service. The blockchains is a public or shared repository containing linked blocks of data. In comparison to all various ledger strategies, blockchains ensure revocability evidence security of authorized activities. according to the shared and distributed structure, blockchains have been used in IoT, - for example, to control network settings, storing device data, and allow multi-payments. It introduces the concept that uses blockchains as an IoT service that examines the efficiency of a blockchain-enabled execution [11]. Figure 2 shows the google trends worldwide[12].

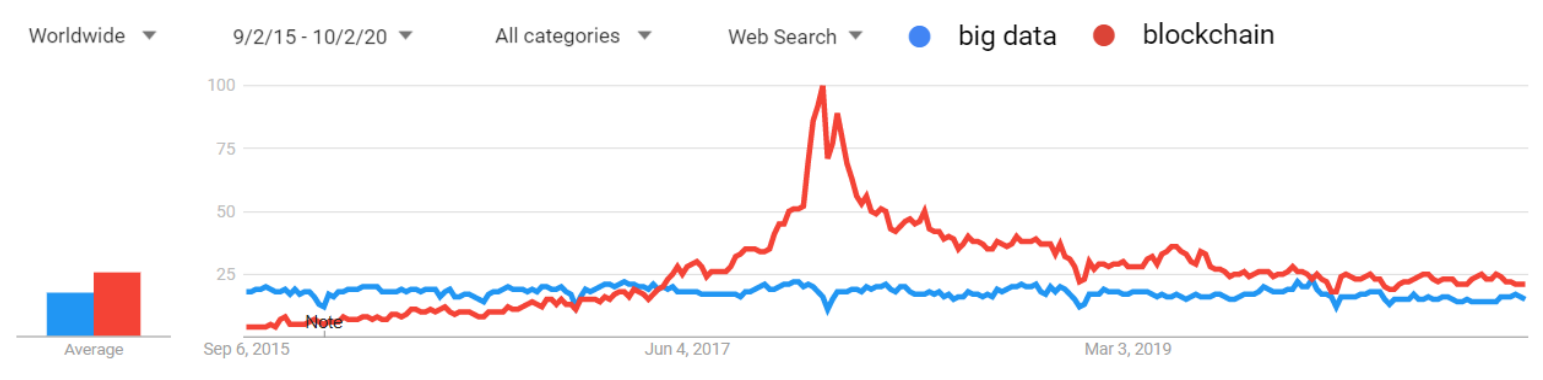

(a) Bigdata vz. Blockchain

compared breakdown by region

- big data blockchain

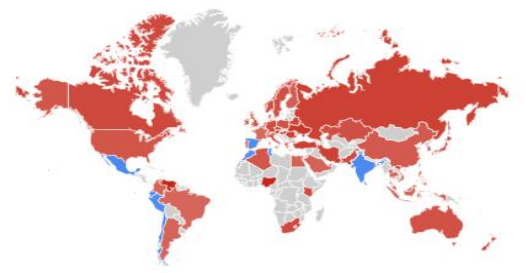

(b) Compared breakdown by regions

Figure 2: (a), (b), Google trends of Bigdata and Blockchain 
In 2017, Ahmad Banafa has published an article on IoT and Blockchain Convergence. He has presented the integration of Blockchain and IoT in this article. Blockchains could be used to monitor billions of smart devices, allowing exchange management and collaboration among connected objects. It enables substantial benefits to IoT technology producers. Such a collaborative approach can remove specific failures, providing a more stable environment for gadgets to operate through. Cryptosystems used throughout blockchains will directly activate information more secure. Blockchains allows secure, peer-to-peer communication permissible and has shown its utility in the context of business transactions via digital currencies, offering assured peer-to-peer payments processing without third-party agents [13].

The primary objective of blockchains is to enable secure communications among parties via a peer-to-peer infrastructure utilizing a shared database. Its purpose is to remove all third - parties verification and replacement the trust of the central authority for verification of transactions for authentication evidence. although several blockchains app revolves through cryptocurrency, blockchains may be used in several other areas, like banking, storage systems, healthcare, robotics, and so on. The blockchains could be used to build distributed apps by building an accessible distributed system [14].

IoT and blockchains are rising in aspects of the advanced social system, and are key consideration societies and cultures, whether individually or combined. Moreover, the use of both techniques for health monitoring to allow open transmission of information between the group members. The features of centrally consolidated and worldwide decentralized measurement are being accessed throughout this blockchain-IoT network by separating others into the base or edge platforms. The whole association improves efficiency and network performance. This same new plan was successful in the experiment of autonomy systems management and surveillance [15]. The blockchains based trust platform for IoT devices is explained in the article [16].

The execution of IoT consists of a moving object of risk requiring authentication measures. Smart devices range through highly scalable situations to business-oriented frameworks. Extensive coverage of IoT protection is required, in particular for highly scalable situations, but related enterprise systems. A variety of security processes and strategies were introduced and used. Blockchains frameworks contribute to protecting several IoT-oriented technologies by being key to the success of a security matrix in the sense of a technique. The blockchain is a ledger that records all evidence activities [17].

This study is a step forward in the architecture of smart cities where we propose a new framework based on blockchain technology and big data analytics. The Blockchain is a decentralized, transparent ledger to store transaction information. It has an amazing ability to create unique structures for most enterprise applications by building trust between nodes effectively. This is a key technology that enables decentralized strategy. The Blockchain has initially captured the attention of the researchers when it emerged as a platform for the secure and efficient exchange of cryptocurrency such as Bitcoin between anonymous people on a public network platform. Presently this innovation generates strong interest to be used in several industries and sectors from business, financing, and insurance to health, government, entertainment, and production. The Blockchain is a kind of notebook or file that includes transaction information. Every transaction generates the hash code. Every block connects to the previous block and brings the Blockchain connected. The Blockchain becomes successful because it is distributed across many devices, each with a Blockchain copy. The Blocks on Blockchain are composed of digital pieces of information. The digital information will be stored in a shared ledger (chain). Even though multiple studies concentrate on the use of Blockchain in numerous application respects but in both technical as well as implementation viewpoints there are very few survey papers found on the Blockchain.

The blockchain-based big data is an approach for secure communication among smart devices using the integration of big data and blockchains. This approach is useful for smart cities. The Blockchain-based big data is becoming a revolutionary invention that operates on a decentralized, distributed, public, and realtime network for collecting activities between IoT nodes [18]. Throughout fact, the blockchain is a series of 
blocks, each block is linked to the previous nodes. Each block will have a public key, a previous block hash, and its metadata [19]. Blockchain-based big data processes would be the specific sections that have been developed to convey data between IoT nodes [20]. Each IoT devices appear to have been various types of potential but smart devices including embedded sensors, cameras, networks, and the ability to communicate with a variety of other IoT nodes [21]. IoT links individuals, sites, objects, or resources for increased efficiency and security. Advanced devices, transceivers are integrated into physical things, every transferring information to the IoT devices. Then IoT analytical tools use this obtained information to transform ideas towards outcomes, improve workflows, and contribute to innovations. Smart cities will deliver the best facilities to promote residents' everyday lives in the areas of medicine, traffic, power use, and skills training. Interestingly, the idea of smart cities is emerging, and, considering its desired future, security measures are on the emergence. blockchains can encourage the growth of smart cities due to its positive characteristics like accountability, openness, flexibility as well as integration.

\section{Possibilities for the proposed integration approach}

The function of Blockchain-based big data will provide an approach to analyzing secure transaction data by IoT nodes [22]. Blockchain-based big data tends to be a comfortable place that may be used both publicly and privately [23]. The connectivity allows the devices to enable the cryptographic operations between IoT nodes under the complicated network [24]. The activities could be tracked and moved anywhere accessible to communicate in the IoT network [25]. The Blockchain tends to be a chain of authenticated or cryptographic blocks controlled by a computer linked to the entire network [26]. Such blocks of data are stored digitally as well as sending data to a blockchain [27]. There are several remarkable possibilities for the integration approach. Figure 3 shows the uses of big data in smart cities.

i) Building trust between the stakeholders: owing to its extensive additional functionality, the Blockchainbased big data approach will establish relationships between numerous wireless technologies. Only verified devices can communicate with the framework since a miner would first verify each block of the transaction, and then connect to the blockchain [28].

ii) Blockchain-based big data technique may reduce costs because it works efficiently without a third - party vendor. It erases all third-party clusters between the sender and the receiver. Such an approach provides great conversation.

iii) Blockchain-based big data helps in reducing a lot of time. Such an approach minimizes transaction time from hours to seconds.

iv) Blockchain-based big data approach ensures privacy and security to devices and information.

v) Blockchain-based big data approach provides connected networks for both social and community facilities. Connected devices can communicate with each other and share data.

vi) This approach can securely exchange money without the use of a third person. It provides rapid, secure, and financial details services. It reduced the transfer time and charge.

vii) This strategy plays a key role in the investigation or elimination of the potential for loss of the asset or activity.

Blockchain-based big data approach may face several challenges, such as scalability, storage, skill levels, and detection, etc.

1. It can be hanged due to the huge transaction volume. Expect that since IoT is integrated with Blockchain, the load will be heavier than that of the current condition.

2. The IoT node will maintain a digital transaction in Blockchain-based big data. During most of the period, it will enhance its processing power, and it will be a challenging task or become a huge load on the connected devices or routers. 


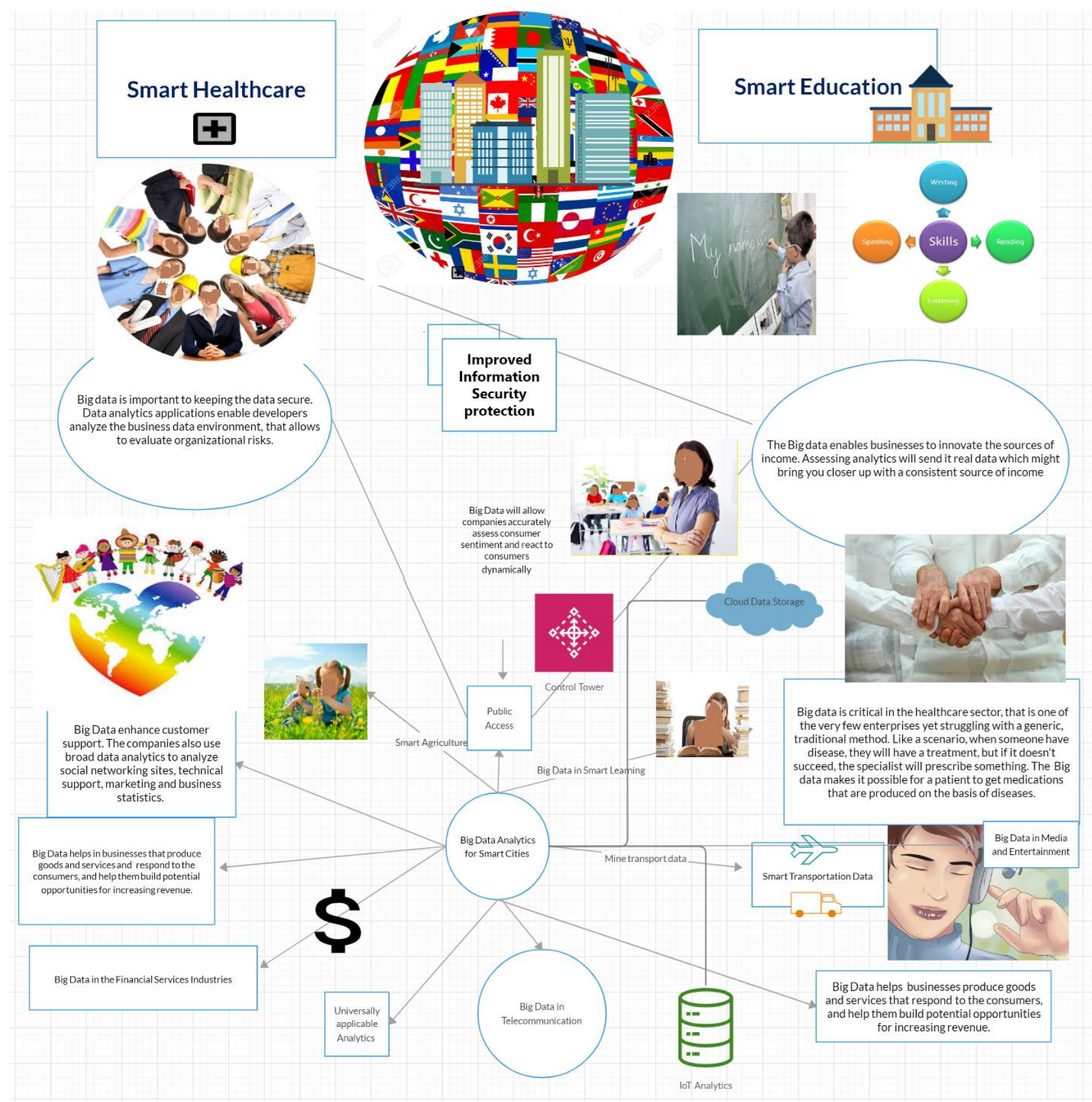

Figure 3. Uses of Big Data in Smart Cities

3. Blockchain has become a radical revolutionary innovation with the IoT. Some people worldwide know this technology.

4. Discovering the nodes is a challenge across all smart devices in this strategy [29]. IoT can discover their connected nodes but when it integrates with the blockchain this task will be challenging [30].

5. The ledger will be freely distributed through all connected nodes [31]. Each node may look at the blockchain activities. Therefore, privacy has been a big challenge in the integrated solution.

6. Blockchain may be either personal or public kind. Interoperability among public and private blockchains would thus also be an obstacle in the integrated approach.

5. Blockchain-based big data will operate globally and is thus faced with several regulations to execute the policy. 
Creating the new model for communication among smart devices in the smart cities is the objective of this research. It is based on blockchain technology with big data, cloud, and Internet of things devices for the smart city. This framework can provide QoS through reducing the traffic rate vacillation also the variety of the number of smart gadgets. We considered the idle state to makes our examination more efficient, at that point, the general execution regarding the overall performance of the framework is evaluated in this research. Blockchain and big data in this framework will monitor and analyze the real-time data collected from IoT nodes and then taking the appropriate decision.

Several countries are planning to adopt the smart city idea in their towns and enforcing large-scale data projects that promote smart city features to achieve the recommended level of sustainable development and enhance standards of living.

The smart city tends to mean an advanced technological area that would be capable of understanding its environment by examining its data so it can make significant changes to solve the problems and improving the quality of life of all its people. The smart cities allow different kinds of wireless sensors to gather massive amounts, full speed and a broad range of city data allow the use of big data technology to learn important observations to control resources, services, and infrastructure effectively. The smart cities use numerous innovations to increase the efficiency of fitness, travel, resources, education, and public infrastructure, resulting in a higher comfort level for their residents. Big data analytics is one of the most recent technologies with great potential to improve smart city facilities. Big data is a huge amount of data collected from different sensors, smart objects, or smart devices connected with the Internet of Things (IoT). Smart cities combine telecommunication technologies and smart devices connected to the IoT system to improve the efficiency of city services and infrastructures and communicate with residents in real-time. Smart cities allow regional officials to communicate directly to both society and city services as well as to track what might be happening in the city or how the city is progressing. Big Data is used to improving the quality, efficiency, and interactivity of metropolitan facilities, lower costs and consumption of resources, and boost interaction between residents and administrations. The applications for smart cities are designed to control residential flows and provide real-time interactions. Smart devices across the world are rising rapidly day by day. Such gadgets provide all consumers with more facilities and connect to everyday lives. The smart devices can effectively connect to the internet for transmitting data inside the area of the service. Connected devices are not always smartphones, these can be smart fridges, smart home technology points of entry, smart air conditioning systems, smart hotspots, smart thermostats, color scheme-changing smart Led lights, smartwatches, smart laptops, and so on.

\section{Methodology and Results}

The success of this research is the development of the Integration system for smart objects using cloud and blockchains on the IoT. The whole structure is mainly designed for applications at which data is accurately transmitted to smart objects on the network of IoT [32], [33]. Also, it is using a re-transmit method, participants' width, or congested traffic problems to strengthen the present framework. The implementation plan for this research is being described. Throughout able to develop content on the internet, the Blockchainbased big data system must be designed to communicate securely among IoT connected systems [34].

This entire analysis will be carried out as a 3-layer architecture, with these layers being clouds, Blockchain, and IoT. Such a study proves data transmission in terms of developing a Blockchain-based big data framework among several smart devices. This architecture has the key sections: a) IoT Nodes b) Internet c) Technology tools d) Blockchains [35]. It creates a basis for accessing secure data documents through IoT nodes. Blockchain exchanges can be controlled or approved by another node confirmed to communicate with the IoT network. IoT-BT can improve communication protection. The main benefits of BT with IoT are: 
1) communication among IoT nodes or minimizing the chance of collisions.

2) to minimize operating costs by having conversations without a third - party.

3) accelerating transaction data.

Many transactions are issued in the permitted blockchain-based file via simulation services, service providers, or cloud holders. The IoT nodes find smart contacts from inside the authorized blockchain-based database [36]. This approved blockchain-based database generates the token for IoT nodes. The IoT node demands its key in the development tools from the remote system and guides the request key [37]. The main system verifies the tokens from an authorize blockchain database or generates a key for each IoT node and system response [38]. An IoT node is enabled to collect data on the network. IoT is becoming an innovation of significant impact throughout many market sectors. Most IoT systems are designed to have worldwide coverage through thousands of easy and often embedded systems. Also, the limited capacities of many IoT devices, and also the existing communications systems focused on centralized frameworks, are posing major issues in the IoT environment [39].

IoT smart devices (D) can be expressed with six tuples as follows.

$\mathrm{D}=\{\mathrm{Id}$, Status, Type, Loc, Spc, O $\}$

Where Id is the unique identification of IoT devices, Status is the status of IoT node, Type is the type of IoT nodes, Loc is the location of IoT node, Spc is the specification and $\mathrm{O}$ is the application object.

Each IoT device has a unique identification number that is generated by the IoT headquarters after entering the device to the network range. The Id is the number on the inherent pattern of the device. The status of the IoT devices can be inactive. Spc could be 0 or 1 where 0 means the device is in an inactive state and 1 means the device is in an active state. The IoT device could sense different types of activity.

Consider the D1, D2 and D3 are three IoT devices, connected in Blockchain-based big data network with the shared hash code H. The Encryption key for D1 is D1E and the Decryption key for D1 is D1D. Consider the D1 device is sending information to D2 and D3. This can be represented as follows [22].

(1) D1 $\rightarrow$ D2: A, H (D1E)

D1 $\rightarrow$ D3: A, H (D1E).

D1 encrypts the D1E key along with hash code $\mathrm{H}$ and sends this information to D2 and D3.

(2) D2 and D3 decrypt H(D1E) and extracts D1E. D2 and D3 will generate 'ACK', encrypts it with H using the D1E key, and sent it to D1.

$\mathrm{D} 2 \rightarrow \mathrm{D} 1: \mathrm{H}(\mathrm{D} 1 \mathrm{E}(\mathrm{ACK}))$

D3 $\rightarrow$ D1: H (D1E (ACK)).

D1 received the information authenticates by D2 and D3, since D2 and D3 can access encryption key D1E from data sent by D1 to D2, D3. However, D2 and D3 should know the hash code H.

(3) D1 decrypts data sent by D2 and D3 and sends the ACK. D1also creates the challenge (ch)D1 and session SD1, then encrypts it using an ACK and sends it to D2, D3.

D1 $\rightarrow$ D2: ACK ((ch)D1, SD1).

D1 $\rightarrow$ D3: ACK ((ch)D1, SD1).

D1 sends ACK ((ch)D1, SD1) to D2 and D3. D2 and D3 can access ACK with hash code H.

(4) D2 decrypts received acknowledgments, access (ch)D1, and SD1. D2 generates hash function h((ch)D1) and ((ch)D2, SD2). So, the complete hash function (h ((ch)D1), (ch)D2, SD2) send to D1, encrypted by ACK acknowledgment.

D2 $\rightarrow$ D1: ACK (h ((ch)D1), (ch)D2, SD2).

D3 decrypts received data, access (ch)D1, and SD1. D3 creates hash function h ((ch)D1) and ((ch)D3, SD3). So, the complete hash function (h ((ch)D1), (ch)D3, SD3) send to D1, encrypted by ACK acknowledgment.

$$
\text { D3 } \rightarrow \text { D1: ACK (h ((ch)D1), (ch)D3, SD3). }
$$


(5) D1 decrypts this information, extracts ((ch)D2, SD2) and ((ch)D3, SD3). D1 process the hash function send by D2 and encrypts ACK acknowledgment. It sends this information to D2. D1 also processes the hash function send by D3 and encrypts the ACK acknowledgment. D1 send it to D3.

$$
\begin{aligned}
& \text { D1 } \rightarrow \text { D2: ACK ((ch)D2) } \\
& \text { D1 } \rightarrow \text { D3: ACK ((ch)D3) }
\end{aligned}
$$

This shows the acknowledgment for D2 and D3 sent by D1.

Every IoT node D1, D2, and D3 know the sessions SD1, SD2, and SD3. These devices can access the key (SD1, SD2, SD3) for sessions generated by D1. Now, they can start communication among D1, D2, and D3. In the experiment, three devices are considered to evaluate the above procedure, However, in the future, we can enhance it for several IoT nodes. After generating the session, the IoT nodes can communicate directly. IoT nodes D1, D2, and D3 are used the challenges (ch)D1, (ch)D2, and (ch)D3 for confirming the secret hash code $\mathrm{H}$ generated by the blockchain. $\mathrm{Al}$ the end of the above procedure the IoT nodes will generate the final session key $\mathrm{S}=(\mathrm{SA}, \mathrm{SB}, \mathrm{SC})$ and distribute to all connected nodes in the network. The following procedure will perform.

$$
\begin{aligned}
& \text { D1 } \rightarrow \text { D2: A, C(D1E), } \\
& \text { D1 } \rightarrow \text { D3: A, C(D1E), and so on. } \\
& \text { D2 } \rightarrow \text { D1: C(D1E (ACK, SD2)). } \\
& \text { D3 } \rightarrow \text { D1: C(D1E (ACK, SD3)). }
\end{aligned}
$$

In (2), the (ch)D2 replaced by SD2, and (ch)D3 was replaced by SD3.

$$
\begin{aligned}
& \text { D1 } \rightarrow \text { D2, D3: ACK(SD1). } \\
& \text { D1 } \rightarrow \text { D2, D3: K(SD1, h(SD1, SD2, SD3)). }
\end{aligned}
$$

In (4), D1 is the sender, and D2, D3 are the receivers.

$$
\begin{aligned}
& \mathrm{D} 2 \rightarrow \mathrm{D} 1: \mathrm{K}(\mathrm{SD} 2, \mathrm{~h}(\mathrm{SD} 1, \mathrm{SD} 2, \mathrm{SD} 3)) \\
& \mathrm{D} 3 \rightarrow \mathrm{D} 1: \mathrm{K}(\mathrm{SD} 3, \mathrm{~h}(\mathrm{SD} 1, \mathrm{SD} 2, \mathrm{SD} 3)) .
\end{aligned}
$$

In (5), D2, D3 are senders and D1 is the receiver.

Data packets are distributed in the cloud by using IoT devices. All blocks have their cryptographic hash, the previous hash block code, and its information in Blockchain. Blockchain does have a link to the sharing of data. Each information contains a lot of blocks including its previous hash code and data of blocks. Those blocks are interconnected with authentication via cryptographic algorithms. Blockchain is like the linked node in the linked list. Its blocks are distributed in a decentralized structure to use a P2P topology framework. If a new block is added, this moves to the system and visits every node connected to the network and confirms its security.

Information transmission between the IoT-devices in the network could pass information from IoTgateways, cloud gateways, blockchain gateways, or other gateways. The other gateways are the sequence of specific gateways other than the IoT-gateways. IoT-gateways is the compilation of gateways among the IoT nodes. Imagine that the interaction delay among IoT nodes is wonder about inconsequential. Think About $\mathrm{t}_{1}$, $t_{2}, t_{3}$, and $t_{4}$ are the transmissions delay functions among the IoT devices to other gateways, other gateways to IoT-gateways, IoT-gateways to cloud gateways, and cloud gateways to blockchain gateways. L1, L2, L3, and L4 are the latencies of other gateways, IoT-gateways, cloud gateways, and blockchain gateways. The data are transmitted from IoT nodes to the other gateways and other gateways to the IoT-gateways then it needs energy consumption. The effectiveness of the proposed method is analyzed via a variety of experimentation. Initially, thousands of blocks of fixed size using open-source software (Node.js) are created and IoT network, linked to the amazon cloud, and create the blockchains network. 
Blockchain-based big data uses smart devices on the network edge of blockchain networks to interact, transfer, and distribute data between IoT nodes. Exchanges in the proposed work have been transmitted to the p2p network. Many special IoT nodes are referred to as Miners in the network. It is typically used in the verification of blockchain networks. If the transactions are processed in such a way that they are converted into blocks or agreed to connect to the current blockchains or transferred to the internet.

The miners have a critical role to play in integrating the development of a new block in the blockchains. The hashing technique is designed using the Hyperledger IROHA method.

Stage 1. Transmission: verify, organize, or register transactions or submit data to the network.

Stage 2: verification and signing: it verifies, queries, or signs the transactions or distributes the verified IoT node of the P2P platform.

Stage 3: linked to the having signed.

The following table 1 shows the transactions using blockchain Ethereum.

Table 1. Blockchain's transactions data

\begin{tabular}{|c|c|c|c|c|c|}
\hline Hashing Code & $\begin{array}{l}\text { Block } \\
\text { no\# }\end{array}$ & From (Device) & To (Device) & Data & Duration \\
\hline 0x28983764646747 ... & 8795992 & 0xhfhj4j5h5h5j5jbkh5jj... & 0xiuufbnfnjjdfjfjfjd4442.... & 0.58497 & 5 secs ago \\
\hline 0xbsyui88777hhghn... & 7859573 & 0xj4m4kk4j4jkk339334... & 0xkjhfgg8877n5bb5b22..... & 0.786533 & 12 secs ago \\
\hline 0x2887hjhdjd09876... & 8575755 & 0xyyfuieg494474j4g5hj... & 0x7llknb5h5j5j5h5h3m1... & 0.7222984 & 10 secs ago \\
\hline 0xk877khh3hh4kkll... & 5333456 & 0x8ahhhdjfjfg48585895... & 0xkjfjjfkj578858599993.... & 0.5673838 & 9 secs ago \\
\hline 0x87fgtrdnhiuye877... & 9098776 & 0x7djhsgsjk98774b4b45... & 0xkj7788y4849nnm44m.... & 0.5588769 & 12 secs ago \\
\hline
\end{tabular}

The hash code is generated when we send data among smart devices. Table 2 shows the hashing of the inputs.

Table 2: Hashing code generation

\begin{tabular}{|c|c|}
\hline Message & Hashing Code \\
\hline Hi, Tanweer & $4667 \mathrm{ee} 0 \mathrm{bb} 829 \mathrm{~d} 7 \mathrm{a} 2 \mathrm{~b} 19164 \mathrm{ee} 4 \mathrm{e} 87 \mathrm{~b} 02 \mathrm{e}$ \\
\hline Faculty of Computer & e36ef5a4f74ff0c14e8668c553363942 \\
\hline Islamic University of Madinah & $5122682 \mathrm{e} 33 \mathrm{~d} 1 \mathrm{~d} 03 \mathrm{db} 41 \mathrm{f} 0 \mathrm{bfee} 6 \mathrm{c} 6 \mathrm{~b} 3 \mathrm{de}$ \\
\hline
\end{tabular}

The hash code will change if we make a small change in it. For example, in table 3, we only change $\mathrm{t}$ as a small letter, but the hash code is changed completely.

Table 3: Hashing code changed if input string change

\begin{tabular}{|c|c|}
\hline Message & Hashing Code \\
\hline Hi, Tanweer & $4667 e e 0 b b 829 d 7 a 2 b 19164 e e 4 e 87 b 02 \mathrm{e}$ \\
\hline Hi, tanweer & 800eee4c6ca52e5b7a3a1a943dd42256 \\
\hline
\end{tabular}

In the case of system failure, the automatic program leads to a step called error tracking. Throughout fact, the technique performs with the current server to detect discrepancies. Study researchers compared the efficiency of 5 and 10 IoT nodes using investigations. The blockchain-based big data system is evaluated with 5 local IoT devices and 2 remote IoT devices. NoSQL database program is used to collect blockchainretrieved information in the cloud of a technology named the OPENSHIFT framework. The cloud is provided by the Amazon server. FogSim technology is being used to link the IoT devices to the network. Blockchain-based big data requires miners in the blockchains network as a result of a study conducted. Initially, two miners were chosen with fixed exchanges as well as establish the nebula specifications with 
miners. Secondly, three miners of specific transactions or established the nebula specifications for miners. Unless the cloud market is rising, the probability of mines the miner's block is stronger. Modeling criteria like the use of a processor, use of storage in blockchains compared with cloud, and edge are tested. The author realized that its use processors in blockchains were smaller than cloud and IoT. Using storage based on the number of blocks and the volume of data. When a block size in the chain increase, storage use may also enhance. Likewise, as the amount of transactions increases, storage use would therefore rise. The author is implemented as an interface in JavaScript that assists the IoT devices to connect with the Blockchainbased big data network. This framework uses the web3 javascript libraries to connect with Ethereum domains via RPC requests and the CoAP JS API,blockchain-based-CoAP 5 to access to sensor nodes.

\section{Discussion}

Smart Cities across the world are getting more efficient. These are introducing policies to foster a greener and healthier urban areas, clean water and air, greater accessibility, and effective social resources. Certain programs are enabled by innovative technology, like the IoT and Communications technologies which provide a technological structure for the implementation of smart city initiatives. The smart cities initiatives require big data analytics to operate. IoT creates large databases that need to be evaluated and stored for the implementation of smart city operations. The Big data networks, building in the smart city infrastructure, should organize, interpret, and filter information obtained from the IoT sensors and devices. Smart Cities government incorporates communication technologies to connect government programs, while at the same moment involving citizens in public government and thereby encouraging collaboration.

The smart city is information-driven through its concept. The Big data analytics perform an increasingly significant role in strategic planning, with certain cities now acting as Community Information Executives in the government. An Integration of big data analytics and smart cities solutions help cities improve the management in critical segments such as big cities have more challenges to manage power usage effectively. Smart city introduction helps city authorities to track energy usages dynamically. Through big data, smart cities can forecast times of high usage and schedule the allocation of power appropriately. IoT enables users to transmit information to blockchains public ledger for participation in public transactions with databases. The Blockchains allows the business participants to connect and distribute IoT data and no need for centralized controlling. The agreement should be checked to avoid conflicts and to ensuring that each participant is kept responsible for certain specific activities. Construct a trustworthy and productive business among individuals and organizations which are working collectively. IoT nodes could engage in exchanges as a decentralized group. Its undeniable records of activities from devices recorded on the blockchain offer evidence of collaboration between companies and individuals. Improve efficiency for the design, management, and compliance of agreements by exchanging data between different groups. The participant in Hyperledger is the IoT device. The agreement is allowed to consent to the status of a distributed public blockchain network. Generate a continuous or indelible database provided by the business and industry policies. Enhance exchanges to make it easier for customers and allow emerging technologies. Smart cities will deliver the best services to improve residents' everyday lives in the areas of medical care, mobility, power usage, and learning. Moreover, the idea of smart cities is still emerging, and, considering its desired future, privacy concerns have been on the emergence. distributed ledger provides the opportunity to facilitate the growth of smart cities due to its positive characteristics like accountability, efficiency, flexibility, and distribution. IoT is shaping the direction of businesses by the utilization of sensor nodes as well as other motion connected technologies. It is a significant strategic initiative that need to preserve knowledge at all stages of the IoT network. For the number of sensors emerging annually, information security is becoming highly complicated. Blockchains can tackle security risks in the embedded network. Blockchains and IoT are integrating throughout all sectors, involving banks, finance, manufacturing, and 
agricultural sectors. Also, in smart homes, suppliers, transportation, and agreements. Agreements connect blockchains to IoT systems to immediately enforce each transaction after certain tasks have been completed. The use of these blockchains helps businesses to process the information on sensor nodes in an IoT environment, decreasing the cost related to mobile device improvement and secure communication. This eliminates the risk of information management since there is no centrally controlled repository of information. Figure 4 shows the performance of the smart device in the proposed integration approach. The author has collected data from various experiments using several factors such as delay by the network, delay by smart device, node energy, mobility energies, execution time, and network usages.

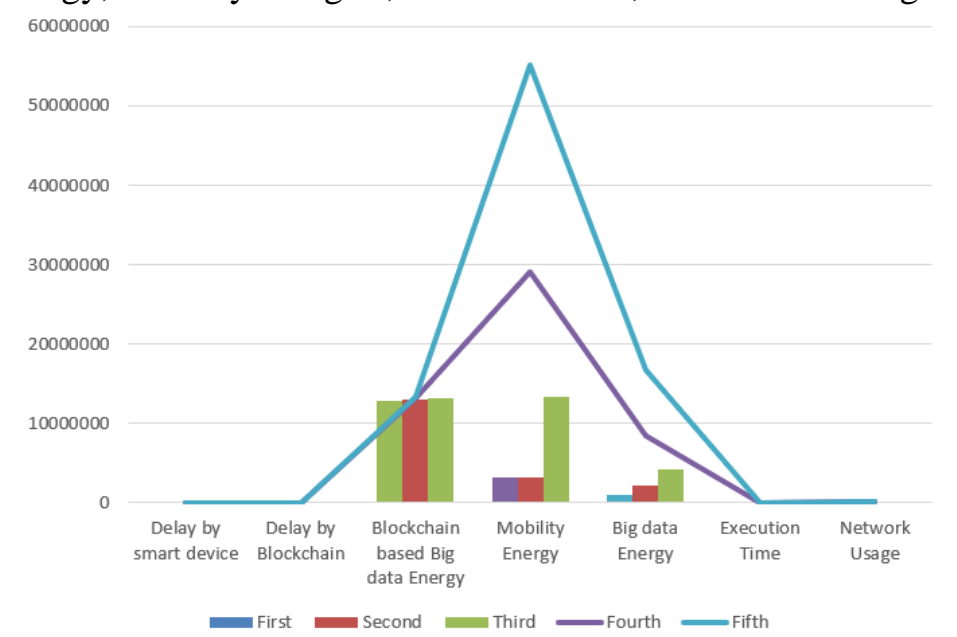

Figure 4: The delay by the network, delay by smart device, node energy, mobility energies, execution time, and network usages

The IoT functions through interacting among smart devices when sending and receiving information that needs internet, mobile networks as well as another way to communicate. Essentially, smart cities are using IoT gadgets to collect and store information effectively for implementation in a specific location. The smart city sensors and smart devices obtain information from various smart city access points configured in a region and then examine it for the great decision-making process. Making sure residents protection has become a primary concern for every community so it is critical to secure citizens in every kind of situation. To prevent future issues inside the area, statistical modeling may help to analyze historic and geographic information to determine whenever and wherever incidents are likely to take place. The substantial amount of success could be the required information to transform the city into something much better location.

\section{Applications of Blockchain-based Big data for Smart cities}

Big data Analytics can collect, analyze as well as mining data about smart city projects in such an intelligent way to generate information to improve various smart city facilities. To turn, big data would enable decision-makers to prepare for any growth of smart city infrastructure, facilities, and places. Smart Cities and Big data applications could be categorized into two groups: disconnected big data apps and Connected big data apps. Connected Big Data apps are distinctive as it depends on immediate feedback and quick evaluation to decide or intervention within a brief as well as the very precise timeframe. In certain instances, when a recommendation could not be reached in the timeframe, it will become meaningless. This is critical that all information required for this assessment is made available promptly and that the study is carried out in a comprehensive and timely manner. As a consequence, big data apps in real-time typically want additional specifications. After designing broad data-based smart city apps, it is important to explain a range of criteria arising from the uniqueness of smart city demands and big data features. The criteria are defined along with the basis of the nature of big data apps and the complexities of developing such apps for 
the smart city. Many of these conditions are technical, whereas others are linked to the resident's knowledge and the function of authorities [40].

\subsection{Smart Learning}

Big Data in the field of education provides excellent possibilities for institutions such as mLearning, curriculum development, enhancing the evaluation process, offering career advice to learners, and introducing new educational strategies [41]. Because of the importance of Big Data in education, organizations may monitor academic achievement along with several disciplines for the students and the global standard and create specific strategies to help learners improve. Data analysis of the scores of learners in numerous topics could assist to direct learners easier and the learning schedules could be applied appropriately. Along with the basis of the success in the corresponding courses and the field of study, educators would be able to advise learners about what direction in life they must pursue. Learners would also be capable of recognizing determinants of the success of students as well as coming up with successful approaches by big data analysis. Many learners can only learn through listening, and many learners could learn through understanding. However, some students may learn through watching the videos and others may use some other techniques. However, some learners are forced to contribute to a given model that may be opposed to their kind of learning techniques, and that is something that discourages their development. It can also have a dramatic effect on the student's educational success. The Big data analytics provide various educational proposals and would then allow learners the right to select their desired thinking strategy that is why there are big data used. Using the means of Big Data Analytics, organizations would be able to construct various learning strategies that will potentially allow them to make it much more enjoyable.

\subsection{Smart Health}

The Big data in health services are an excellent fit for this. This enhances the health sector more than before. The volume of data that the health industry must concern with would be overwhelming. The days have gone when medical providers have been unable to take advantage of such information. Including seeking a cure from diseases, the Big Data has brought everything under its umbrella, and investigators have seen too many lives-saving results. Connected wearable technology recently continued to gain prominence and are the current trends across individuals of all age categories. It creates huge quantities of real-time data in the context of warnings that help to save people's lives [42].

\subsection{Smart Tourism}

The Big data could help humans in the tourism industries in a variety of many respects, helping everyone to reach better proof-driven choices. This allows predicting potential activity efficiently, to refine business models, to sell information better specifically, and to enhance customer satisfaction. Smart Cities have had an enormous wealth of experience which can change the future of tourists. But merely getting knowledge does not have to be prepared to do so efficiently. Smart Cities and travel participants would need access to services that will allow them to derive implementable inferences. Several of these findings would allow them to support not only tourists, as well as locals [43].

\subsection{Smart retail economy}

Modern technologies have also been a mechanism for socially and economically transformations. Renewable technologies, innovations, and communication channels have brought great changes to the community, to the financial system, and citizens during the recent economic booms. That is not different today. The current economic model of the digital economy is now the basis for commercial activity. People begin experiencing the emergence of a modern technology era. The latest smart ecosystem has an 
unparalleled effect, which is both overwhelming and satisfying. A retail sector is facing the most extreme rivalry among everyone. Dealers are continually looking for forms that offer businesses a strategic advantage over anyone else. Consumers have always been, in fact, the true winner of sensations for the retail sector. For distributors to succeed throughout this dynamic environment, they will have to understand better potential consumers. When they understand the expectations of their clients as well as how to better satisfy their expectations, they understand best [44].

\subsection{Media and Entertainment}

The Big data in the media and entertainment sector also often allows companies to achieve confidential information through consumer conduct, as well as helps produce customized advertising. The Big data thus held the key to making entertainment and media businesses more competitive. Media and entertainment organizations would use big data to determine which user information, goods, and apps they need. Due to the study of consumer information, software changes became more economically efficient and timely efficient. They would never realize that functionality the customers need or want to deliver when they don't look into the details, it might offer them a significant advantage, increase market share and customer satisfaction [45].

\subsection{Telecommunication}

Telecommunications companies are gathering vast quantities of data from phone history reports, cellphone uses, internet infrastructure, database records, bills, and social networking sites, offering a lot of details regarding their consumers and the infrastructure, and how telecommunications companies could use this information to enhance their company. Industries with Big Data could monitor areas with the minimum and maximum data traffic rates but also do what is needed to ensure trouble-free network access. The Big Data, as well as other sectors, have allowed the telecommunications industry to recognize its consumers relatively effectively. Telecommunications companies are now supplying consumers with the most personalized services available. The Big Data would be behind the digital explosion that we are now witnessing. Global telecommunications industries are the essence of the industrial revolution taking place over time. To the ever-increasing popularity of smartphones, the telecommunications company has been overwhelmed with huge amounts of storage. Using methods of Big Data Analytics, firms are willing to have consumers with seamless access, thereby removing all the infrastructure obstacles that consumers are faced with [46].

\subsection{Automobile Industries}

Big Data has now turned their back on but are in complete charge of the guiding of the automotive sector. This idea of smart devices is not only restricted to wearable devices but has been at the core of automotive manufacturing. Besides the IT sector, the automobile industry has benefited most from the Big Big data. This is revolutionized the way all pass about. There is an opportunity for autonomous vehicles to make our roads safer, as $90 \%$ of the number of deaths on the highways is due to the mistakes of humans. Those automobiles require information to be genuine. Vehicles become completely fitted with sensors that monitor everything including location, distance, movement, and steering, traffic lights, traffic danger as well as emergencies. Through making use of certain information, the automobile can infer and carrying out possible responses without human mistakes [47].

\subsection{Financial Services Industries}

The Big data is a common new slogan in the field of computing and analytical approaches for gathering and analyzing vast quantities of data. Since before Big Data came to prominence, the financial sector had already dominated the technological sector. The financial institutions were among the first to embrace Big Data. Advancements in computer resources, together with dropping costs, are making massive information 
initiatives progressively technologically and technically feasible. To general, the emergence of cloud services puts the expense of large amounts of data processing beyond the scope of several smaller companies that do not need to make major reinvestment in their technological assets. Its functionality of every financial institution depends greatly on its information and maintaining the information is one of the most challenging tasks facing any financial company. Information was the second biggest resource after money for everyone. Internet banking and transactions are one of the trendiest phrases and Big Data seems to be at the center of this one. The Big Data is responsible for important areas of financial companies including fraud prevention, risk management, statistical arbitrage, and service and support [48].

\section{Conclusion and Future Scope}

This research approach is developed and constructed using IoT, clouds, and blockchains. It is an integration of IoT and Blockchains. Blockchains technology is used to generate a highly distributed public realistic database for transaction processing. Analysis has created an entirely new possibility throughout this field. A methodology is designed and evaluated to use a wide variety of IoT devices. This research could be a useful tool for improving the efficiency of the IoT system in an interconnected context. The method is designed for the provision of interaction security where big data are exchanged in a complex context in the long run. Researchers have evaluated the system in several situations, like memory and storage use in the integrated system, as well as its effect on the secure operation. Researchers find that the proposed method not only improves the performance but also removes the direct relation between IoT nodes, which makes this method more reliable. Its research findings set out an innovative IoT framework with blockchains.

\section{References}

[1] Nawaratne, Rashmika, Damminda Alahakoon, Daswin De Silva, Prem Chhetri, and Naveen Chilamkurti. "Selfevolving intelligent algorithms for facilitating data interoperability in IoT environments." Future Generation Computer Systems 86 (2018): 421-432.

[2] Alam, Tanweer, and Mohammed Aljohani. "Design and implementation of an Ad Hoc Network among Android smart devices." In 2015 International Conference on Green Computing and Internet of Things (ICGCIoT), pp. 1322-1327. IEEE, 2015.

[3] Tanweer Alam. "IoT-Fog: A Communication Framework using Blockchain in the Internet of Things.", International Journal of Recent Technology and Engineering (IJRTE). Vol. 7(6), 2019.

[4] Tanweer Alam, Mohammad Ayoub Khan, Natheer K. Gharaibeh, and Malik Khlaif Gharaibeh, "Big Data for Smart Cities: A Case Study of NEOM City, Saudi Arabia", Smart Cities: A Data Analytics Perspective, Lecture Notes in Intelligent Transportation and Infrastructure, https://doi.org/10.1007/978-3-030-60922-1_11

[5] Tanweer Alam. Shamimul Qamar. Amit Dixit. Mohamed Benaida. "Genetic Algorithm: Reviews, Implementations, and Applications.", International Journal of Engineering Pedagogy (iJEP). 2020.

[6] Tanweer Alam, Mohamed Benaida, "Blockchain, Fog and IoT Integrated Framework: Review, Architecture and Evaluation”, Technology Reports of Kansai University, Volume - 62, Issue 02, 2020.

[7] Karafiloski, Elena, and Anastas Mishev. "Blockchain solutions for big data challenges: A literature review." In IEEE EUROCON 2017-17th International Conference on Smart Technologies, pp. 763-768. IEEE, 2017.

[8] Tanweer Alam. Mohamed Benaida. "CICS: Cloud-Internet Communication Security Framework for the Internet of Smart Devices.", International Journal of Interactive Mobile Technologies (iJIM). Vol 12(6). 2018. DOI: $10.3991 /$ ijim.v12i6.6776.

[9] Tanweer Alam. "A Reliable Framework for Communication in Internet of Smart Devices using IEEE 802.15.4.", ARPN Journal of Engineering and Applied Sciences. Vol 13(10). 2018.

[10] Metcalfe, Robert M. Packet communication. No. MAC-TR-114. Massachusetts Inst of Tech Cambridge Project Mac, 1973.

[11] Samaniego, Mayra, Uurtsaikh Jamsrandorj, and Ralph Deters. "Blockchain as a Service for IoT." In 2016 IEEE international conference on internet of things (iThings) and IEEE green computing and communications 
(GreenCom) and IEEE cyber, physical and social computing (CPSCom) and IEEE smart data (SmartData), pp. 433-436. IEEE, 2016.

[12] Google Trends: 02\&q=big\%20data,blockchain

https://trends.google.com/trends/explore?date=2015-09-02\%202020-10-

[13] Banafa, Ahmed. "IoT and blockchain convergence: benefits and challenges." IEEE Internet of Things (2017).

[14] Florea, Bogdan Cristian. "Blockchain and Internet of Things data provider for smart applications." In 2018 7th Mediterranean Conference on Embedded Computing (MECO), pp. 1-4. IEEE, 2018.

[15] Jo, Byung Wan, Rana Muhammad Asad Khan, and Yun-Sung Lee. "Hybrid blockchain and internet-of-things network for underground structure health monitoring." Sensors 18, no. 12 (2018): 4268.

[16] Di Pietro, Roberto, Xavier Salleras, Matteo Signorini, and Erez Waisbard. "A blockchain-based Trust System for the Internet of Things." In Proceedings of the 23nd ACM on Symposium on Access Control Models and Technologies, pp. 77-83. 2018.

[17] Minoli, Daniel, and Benedict Occhiogrosso. "Blockchain mechanisms for IoT security." Internet of Things 1 (2018): 1-13.

[18] Dai, Hong-Ning, Zibin Zheng, and Yan Zhang. "Blockchain for Internet of Things: A survey." IEEE Internet of Things Journal 6, no. 5 (2019): 8076-8094.

[19] Wang, Xu, Xuan Zha, Wei Ni, Ren Ping Liu, Y. Jay Guo, Xinxin Niu, and Kangfeng Zheng. "Survey on blockchain for Internet of Things." Computer Communications 136 (2019): 10-29.

[20] Conoscenti, Marco, Antonio Vetro, and Juan Carlos De Martin. "Blockchain for the Internet of Things: A systematic literature review." In 2016 IEEE/ACS 13th International Conference of Computer Systems and Applications (AICCSA), pp. 1-6. IEEE, 2016.

[21] Tanweer Alam, Mohamed Benaida. "Blockchain and Internet of Things in Higher Education." Universal Journal of Educational Research. 8.5 (2020) 2164 - 2174. doi: 10.13189/ujer.2020.080556.

[22] Alam, Tanweer, and Mohammed Aljohani. "An approach to secure communication in mobile ad-hoc networks of Android devices." In 2015 International Conference on Intelligent Informatics and Biomedical Sciences (ICIIBMS), pp. 371-375. IEEE, 2015. DOI: https://doi.org/10.1109/iciibms.2015.7439466

[23] Alphand, Olivier, Michele Amoretti, Timothy Claeys, Simone Dall'Asta, Andrzej Duda, Gianluigi Ferrari, Franck Rousseau, Bernard Tourancheau, Luca Veltri, and Francesco Zanichelli. "IoTChain: A blockchain security architecture for the Internet of Things." In 2018 IEEE wireless communications and networking conference (WCNC), pp. 1-6. IEEE, 2018.

[24] Christidis, Konstantinos, and Michael Devetsikiotis. "Blockchains and smart contracts for the internet of things." Ieee Access 4 (2016): 2292-2303.

[25] Jiang, Tigang, Hua Fang, and Honggang Wang. "Blockchain-based internet of vehicles: Distributed network architecture and performance analysis." IEEE Internet of Things Journal 6, no. 3 (2018): 4640-4649.

[26] Tanweer Alam. "Blockchain and its Role in the Internet of Things (IoT).", International Journal of Scientific Research in Computer Science, Engineering and Information Technology. Vol 5(1), 2019. DOI: 10.32628/CSEIT195137

[27] Alam, Tanweer, and Mohamed Benaida. "The Role of Cloud-MANET Framework in the Internet of Things (IoT)." International Journal of Online Engineering 14, no. 12 (2018).

[28] Teslya, Nikolay, and Igor Ryabchikov. "Blockchain-based platform architecture for industrial IoT." In 2017 21st Conference of Open Innovations Association (FRUCT), pp. 321-329. IEEE, 2017.

[29] Yang, Yanhan, Yaming Yang, Jinlian Chen, and Mingzhe Liu. "Application of blockchain in internet of things." In International Conference on Cloud Computing and Security, pp. 73-82. Springer, Cham, 2018.

[30] Mo, Bing, Kuiren Su, Songjie Wei, Cai Liu, and Jianping Guo. "A solution for internet of things based on blockchain technology." In 2018 IEEE International Conference on Service Operations and Logistics, and Informatics (SOLI), pp. 112-117. IEEE, 2018.

[31] Mercuri, Marc E., Zeyad Rajabi, and Eric I. Maino. "Internet of things blockchain interface." U.S. Patent Application 15/995,078, filed January 10, 2019.

[32] Zhao, Shanshan, Shancang Li, and Yufeng Yao. "Blockchain enabled industrial Internet of Things technology." IEEE Transactions on Computational Social Systems 6, no. 6 (2019): 1442-1453. 
[33] Casado-Vara, Roberto, Fernando de la Prieta, Javier Prieto, and Juan M. Corchado. "Blockchain framework for IoT data quality via edge computing." In Proceedings of the 1st Workshop on Blockchain-enabled Networked Sensor Systems, pp. 19-24. 2018.

[34] Rakovic, Valentin, Jovan Karamachoski, Vladimir Atanasovski, and Liljana Gavrilovska. "Blockchain Paradigm and Internet of Things." Wireless Personal Communications 106, no. 1 (2019): 219-235.

[35] Choudhary, T., C. Virmani, and D. Juneja. "Convergence of Blockchain and IoT: An Edge Over Technologies." In Toward Social Internet of Things (SIoT): Enabling Technologies, Architectures and Applications, pp. 299316. Springer, Cham, 2020.

[36] Alam, Tanweer. "Middleware Implementation in Cloud-MANET Mobility Model for Internet of Smart Devices", International Journal of Computer Science and Network Security, 17(5), 2017. Pp. 86-94

[37] Urmila, M. S., Balaji Hariharan, and Rekha Prabha. "Enabling Internet of Things (IoT) Security via Blockchain Framework." In Innovations in Computer Science and Engineering, pp. 575-582. Springer, Singapore, 2020.

[38] Pavithran, Deepa, Khaled Shaalan, Jamal N. Al-Karaki, and Amjad Gawanmeh. "Towards building a blockchain framework for IoT." Cluster Computing (2020): 1-15.

[39] Novo, O. (2018). Blockchain meets IoT: An architecture for scalable access management in IoT. IEEE Internet of Things Journal, 5(2), 1184-1195.

[40] Al Nuaimi, Eiman, Hind Al Neyadi, Nader Mohamed, and Jameela Al-Jaroodi. "Applications of big data to smart cities." Journal of Internet Services and Applications, Vol. 6, no. 1 (2015): 25.

[41] Hammad, Rawad, and David Ludlow, "Towards a smart learning environment for smart city governance", In Proceedings of the 9th International Conference on Utility and Cloud Computing, pp. 185-190. 2016.

[42] Pramanik, Md Ileas, Raymond YK Lau, Haluk Demirkan, and Md Abul Kalam Azad. "Smart health: Big data enabled health paradigm within smart cities", Expert Systems with Applications, 87 (2017): 370-383.

[43] Xiang, Zheng, and Daniel R. Fesenmaier, "Big data analytics, tourism design and smart tourism" In Analytics in smart tourism design, pp. 299-307. Springer, Cham, 2017.

[44] Bradlow, Eric T., Manish Gangwar, Praveen Kopalle, and Sudhir Voleti, "The role of big data and predictive analytics in retailing", Journal of Retailing 93, no. 1 (2017): 79-95.

[45] Schlieski, Tawny, and Brian David Johnson, "Entertainment in the age of big data" Proceedings of the IEEE 100, no. Special Centennial Issue (2012): 1404-1408.

[46] Wang, Zhen, Guofu Wei, Yaling Zhan, and Yanhuan Sun, "Big data in telecommunication operators: data, platform and practices", Journal of Communications and Information Networks, vol 2, no. 3 (2017): 78-91.

[47] Lv, Sijin, "Design of the Automobile Marketing System Based on the Big Data", In International conference on Big Data Analytics for Cyber-Physical-Systems, pp. 1713-1719. Springer, Singapore, 2019.

[48] Fang, Bin, and Peng Zhang, "Big data in finance", In Big data concepts, theories, and applications, pp. 391-412. Springer, Cham, 2016. 